\title{
LARVAS DE Anisakis physeteris Y OTROS HELMINTOS EN Coryphaena hippurus "PERICO" COMERCIALIZADOS EN EL MERCADO PESQUERO DE VENTANILLA, CALLAO, PERÚ
}

\section{Anisakis physeteris LARVAE AND OTHERS HELMINTHS IN Coryphaena hippurus "PERICO” SOLD IN VENTANILLA FISHERIES MARKET, CALLAO, PERU}

\author{
Rufino Cabrera', Luis Suárez-Ognio', Rosa Martínez², Rene Leiva ${ }^{3}$, Carlos \\ Gambirazio ${ }^{1}$, Julio Ruiz ${ }^{1}$
}

\begin{abstract}
Resumen
El objetivo de la investigación fue determinar la prevalencia e intensidad media de infección por larvas del nemátode Anisakis physeteris y otros helmintos según talla y sexo. El estudio se realizó en 57 especímenes de Coryphaena hippurus seleccionados al azar en el Mercado Pesquero del distrito de Ventanilla, Callao, Perú, en mayo de 2002. La prevalencia e intensidad media para larvas de $A$. physeteris fueron de $33,33 \%$ y 1,37 ; para larvas y adultos de Hysterotylacium sp., fueron $100 \%$ y 88,93 ; para metacestodes Tentacularia coryphaenae fue $71,92 \%$ y 6,44; para Nybelinia sp. fueron 19,29\% y 2,64; para los tremátodes Bathycotyle coryphaenae fueron $28,07 \%$ y 2,25 y para Dinurus spp., fueron $22,81 \%$ y 3,42 , respectivamente. Los peces cuyo tamaño está entre $127-130 \mathrm{~cm}$ son los más parasitados por larvas de $A$. physeteris. Esta especie es prevalente en la población evaluada y a pesar de la baja intensidad media, existe riesgo de adquirir anisakidosis si se consume C. Hippurus insuficientemente cocido o crudo.
\end{abstract}

Palabras clave: Coryphaena hippurus, helmintos, Anisakis physeteris, anisakidosis, Perú

\begin{abstract}
The aim of this study was to determine the prevalence and mean intensity of infection by the nematode Anisakis physeteris larvae and other helminths according to size and sex. The study was performed on 57 specimens of Coryphaena hippurus selected at random in "Ventanilla" Fisheries Market, Callao, Peru in May 2002. The prevalence and the mean intensity to A. physeteris larvae were of $33,33 \%$ and 1,37 ; to Hysterotylacium sp., adult and larvae were $100 \%$ and $88,93 \%$; to Tentacularia coryphaenae metacestods were $71,92 \%$ and 6,44; to Nybelinia sp., were 19,29 and 2,64; to Bathycotyle coryphaenae trematods were $28,07 \%$ and 2,25 and to Dinurus spp., were $22,81 \%$ and 3,42 , respectively. The fishes between $127-130 \mathrm{~cm}$ of size were the most parasitized by $A$. physeteris larvae. This species was prevalent in the evaluated population and in spite of the low mean intensity, there is a risk to contract anisakidosis if raw or uncooked C. hippurus is eaten.
\end{abstract}

Keywords: Coryphaena hippurus, helminths, Anisakis physeteris, anisakidosis, Peru.

(1) Oficina General de Epidemiología, Ministerio de Salud. Camilo Carrillo 402, Lima 11, Lima, Perú. Telefax: 433-0081. E-mail: rcabrerach@hotmail.com

(2) Laboratorio de Parasitología, Facultad de Ciencias Biológicas, Universidad Nacional Mayor de San Marcos. Av. Venezuela s/n, Lima, Perú.

(3) Oficina de Epidemiología, Dirección de Salud del Callao. Jr. Colina s/n, Bellavista, Callao, Perú.

\section{Introducción}

El conocimiento de los aspectos ecológicos de helmintos en especies marinas de interés comercial de consumo directo que afectan la salud humana es importante para determinar la magnitud de la infección y proponer algunas medidas de control. Entre estos patógenos están las larvas de nemátodes Anisakis simplex, A. physeteris y Pseudoterranova decipiens que ocasionan una zoonosis para- 
sitaria denominada anisakidosis, caracterizada por una infiltración eosinofílica, que evoluciona hasta la formación de granulomas debido a la ingesta de potajes crudos o insuficientemente cocidos de peces marinos o calamares infectados con estas larvas (Kagei et al., 1978; Asato et al., 1991; Ishikura et al., 1992). Los adultos parasitan a mamíferos marinos (ballenas, delfines y lobos marinos). El hombre es un huésped paraténico porque la larva no se desarrolla hasta el estadio adulto.

En el Perú se han comunicado dos casos por larvas de $P$. decipiens, por haber ingerido “cebiche” en Lima (Tantaleán y Huiza, 1993), y un caso de localización gástrica aguda por larvas de Anisakis sp., en un consultorio privado en Lima (Barriga et al., 1999). En la ciudad de Ica, se han reportado dos casos probables de la forma gástrica aguda por larvas de A. physeteris, y se ha postulado que es emergente durante el fenómeno "El Niño". Además, la prevalencia e intensidad media por larvas de $A$. physeteris en 12 especímenes de Coryphaena hippurus "perico" o "dorado" comercializados en los mercados fue elevada (Cabrera y Suárez-Ognio, 2002). Por esta razón, se realizó el presente estudio con el objetivo de determinar y analizar la prevalencia y la intensidad media de infección por larvas de A. physeteris y otros helmintos en $C$. hippurus de acuerdo al sexo y la talla, comercializados en el Mercado Pesquero del distrito de Ventanilla, Callao, en la costa central del Perú.

\section{Materiales y métodos}

\section{Población}

Estuvo constituida por 136 "pericos", capturados el 07 y 18 de mayo de 2002, mediante el arte de espinel entre 100-120 millas marinas frente a las costas de Paita, Piura, desembarcados en el Terminal Pesquero de Paita, transportados para su comercialización en una cámara isotérmica vía terrestre al Mercado Pesquero de Ventanilla, Callao.

\section{Muestra y selección}

La muestra constituida por 57 especímenes fue calculada mediante la fórmula para una población finita, considerando una prevalencia hipotética de 0,21 con un nivel de confianza de $95 \%$ y una precisión de $\pm 0,08$. La selección de los "pericos" fue mediante un muestreo aleatorio simple. La unidad de muestreo fue la cámara isotérmica y la unidad de análisis el "perico" completo, fresco y sin eviscerar.

\section{Variables investigadas}

La talla se determinó con un instrumento en $\mathrm{mm}$ midiendo cada espécimen desde el extremo distal de la cola caudal al extremo distal de la boca, y para identificar el sexo se realizó la lectura macroscópica de las gónadas. Las definiciones de prevalencia e intensidad media aplicadas fueron las que han sido propuestas por Bush et al. (1997).

\section{Procedimientos y técnicas parasitológicas}

Las larvas y adultos de helmintos se buscaron en branquias, cavidad bucal, superficie visceral y en la musculatura somática. Los contenidos intestinales y estomacales fueron tamizados y sedimentados en forma individual. Las larvas de nemátodes se fijaron en alcohol caliente al 70\%; los metacéstodes y tremátodes adultos, en formol caliente al 10\%. Las larvas de nemátodes se identificaron aclarándolas en una mezcla de alcohol-fenol y comparándolas con la ayuda de la clave de la familia Anisakidae propuesta por Koyama et al. (1969). Los metacéstodes y tremátodes se colorearon por el método de carmín acético de Semichon para su identificación. Los tremátodes se identificaron hasta género y especie, según Tantaleán et al. (1992).

\section{Registro de datos y análisis estadísticos}

Los datos fueron recogidos en un instrumento (ficha); luego, se elaboró una base de datos y se analizaron mediante la ayuda del software EPI INFO (versión 6.4) y para la presentación de los resultados se hizo un análisis univariado y bivariado. 


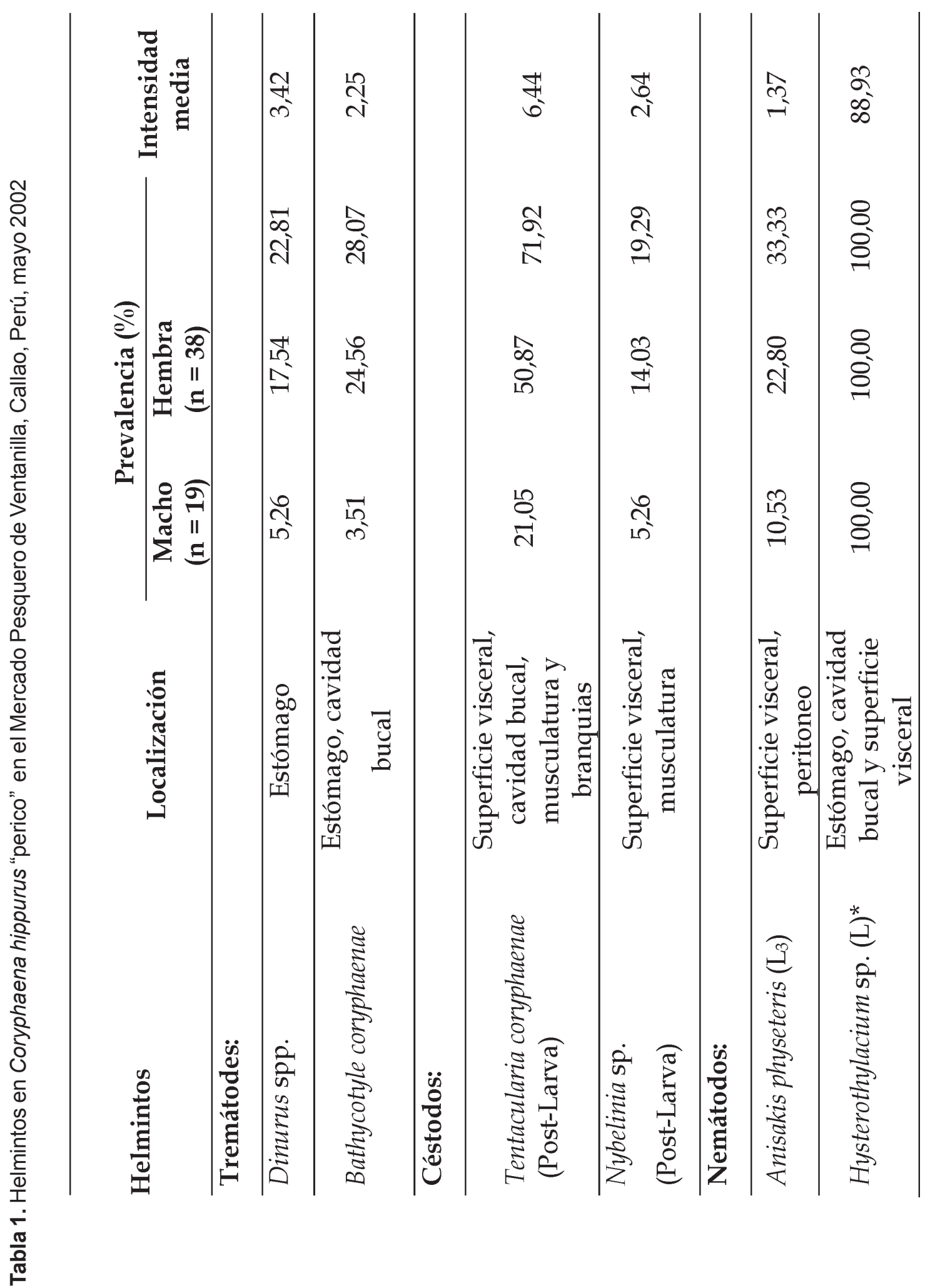


Tabla 2. Prevalencia e intensidad media de larvas de Anisakis physeteris por talla en Coryphaena hippurus en el Mercado Pesquero de Ventanilla, Callao, Perú. Mayo 2002.

\begin{tabular}{|c|c|c|c|}
\hline \multirow{2}{*}{$\begin{array}{l}\text { Talla } \\
\text { (cm) }\end{array}$} & \multicolumn{2}{|c|}{ Prevalencia } & \multirow{2}{*}{$\begin{array}{c}\text { Intensidad } \\
\text { media }\end{array}$} \\
\hline & N. & $\%$ & \\
\hline $119-122$ & 3 & 5,26 & 1,00 \\
\hline $123-126$ & 3 & 5,26 & 2,66 \\
\hline $127-130$ & 9 & 15,79 & 1,11 \\
\hline $131-134$ & 2 & 3,51 & 1,00 \\
\hline $135-138$ & 2 & 3,51 & 1,5 \\
\hline Total & 19 & 33,33 & 1,37 \\
\hline
\end{tabular}

\section{Resultados}

La prevalencia por especies de helmintos, según el sexo en machos y hembras de $C$. hippurus y la intensidad media para nemátodes de la familia Anisakidae, fue $A$. physeteris $33,33 \%, 10,53 \%$ y $22,80 \%$ y 1,37 , respectivamente; para larvas y adultos de Hysterothylacium sp., 100\% en ambos sexos y 88,93 de intensidad media; para tremátodes: Dinurus spp., 22,81\%, en machos 5,26\% y en hembras $17,54 \%$ y la intensidad media 3,42 ; para B. coryphaenae $28,07 \%, 10,53 \%$ y $36,84 \%$ y 2,25 , respectivamente; para metacéstodes de T. coryphaenae 71,92\%, $21,05 \%$ y $50,87 \%$ y 6,44 , respectivamente y para Nybelinia sp., 19,29\%, 5,26\% en machos y $14,03 \%$ en hembras y una intensidad media de 2.64 (Tabla 1).

Los peces cuyo tamaño está entre 127-130 cm son los más parasitados $(15,79 \%)$ por larvas de $A$. physeteris. El promedio de larvas (intensidad media) de $A$. physeteris más alto se halló en los "pericos" entre 123-126 cm con 2,66 parásitos, seguido de especímenes que medían entre 135-138 cm (Tabla 2).

\section{Discusión}

La prevalencia e intensidad media de infección por larvas de A. physeteris, única especie patógena para el hombre hallada en el presente trabajo, fue baja comparada con un estudio previo (Cabrera y Suárez-Ognio, 2002). Las hembras fueron más afectadas que los machos. Esta discrepancia se explicaría por las diferencias en la época del estudio; el nuestro se realizó en mayo de 2002 en condiciones aparentemente normales, a diferencia de los autores mencionados que examinaron el caso durante el "El Niño" en 1997. También estaría relacionada con la migración y procedencia de las poblaciones de "pericos". La prevalencia es menor a la hallada en Scomber japonicus "caballa" en las costas de Pisco, Ica (Cabrera y Tantaleán, 1994), Sarda sarda chilensis "bonito" y Trachurus murphyi "jurel" comercializados en el Callao (Pérez et al., 1999). Las tallas mayores son las más parasitadas, y el consumo de "pericos" de tamaño grande podría incrementar el riesgo de infección. La relación entre la talla y la infección fue sugerida para S. japonicus "caballa" (Cabrera y Tantaleán, 1995). La dinámica de infección actual por larvas de 
Anisakis en peces de la costa peruana son desconocidas. Hace 30 años el "jurel” era la especie más parasitada en el Callao (Tantaleán, 1972); sin embargo, no tenemos argumentos válidos para explicar estas diferencias, aunque es posible especular que se debe a los cambios climáticos producidos en el ecosistema marino. A diferencia de $A$. physeteris, la prevalencia de $A$. simplex en otras latitudes varía, y puede alcanzar hasta el $100 \%$ en peces teleósteos de las costas de Noruega (Strømnes et al., 2000); en la Unión de Emiratos Árabes parasita a 40 especies comerciales, en algunos casos asociadas a Hysterothylacium sp. (Kardousha, 1992).

Los resultados sólo podrían inferirse respecto de los "pericos" procedentes de Paita; por lo tanto, la población que consume este recurso (Ventanilla y otros distritos del Callao y posiblemente Lima y Paita) estaría expuesta al riesgo de infectarse, pero podría variar en función de la dinámica de infección de la población de "pericos" y de la estacionalidad. C. hippurus es una especie oceánica de aguas calientes con mayor volumen de desembarque durante El Niño (Estrella et al., 1998), y el incremento del consumo de "cebiche" podría incrementar el riesgo de infección. La magnitud de la infección en humanos se puede conocer por estudios transversales, pero considerando la presentación de un probable fenómeno de "El Niño" puede detectarse los casos a través de una vigilancia centinela. Los estudios longitudinales de la infección por estas larvas en peces comerciales pueden dar algunas luces para plantear adecuadas medidas de control.

La prevalencia e intensidad media de Hysterothylacium sp., helmintos no patógenos para el hombre, es superior a los hallazgos en Ica (Cabrera y Suárez-Ognio, 2002), que podría deberse al método de diagnóstico usado en nuestro estudio. Su frecuencia de acuerdo al sexo no mostró diferencias; Sarda sarda chilensis "bonito" en el Callao está parasitada por larvas de H. aduncum (Pérez et al., 1999);
Dinurus spp. es más frecuente en las hembras, se han reportado 3 especies en este hospedero (Ruelas y Córdova, 1993), la prevalencia de $B$. coryphaenae es más baja que la hallada en la ciudad de Trujillo (Barrantes y Jara, 1992) y más frecuente en las hembras, que estaría relacionada con su comportamiento. Las frecuencias de metacéstodes de $T$. coryphaenae y Nybelinia sp. difieren de los hallazgos previos, y sería probablemente una de las causas del rechazo del consumo por la población.

Con respecto a las estrategias de prevención y control de los parásitos de importancia médica e ictiopatológica, debe evaluarse la evisceración del pescado antes de ser congelado por los pescadores artesanales, como posible estrategia para reducir el riesgo de infección humana (Cabrera y Suárez-Ognio, 2002), hasta ahora, podría ser una alternativa de control adecuado no sólo contra los de importancia médica sino también contra los de interés ictiopatológico.

\section{Agradecimientos}

Deseamos agradecer a la Dirección de Salud Callao, Ministerio de Salud, por el apoyo para este proyecto; así mismo, a los estadísticos Lic. Luis Roldán y Kennedy Alva, de la Oficina General de Epidemiología, Ministerio de Salud, por el apoyo en el análisis y diseño; a la Blga. Zoila Pizarro y Téc. Alfonso Acosta de la Dirección de Salud Callao; a los Bachilleres Eddie Barrantes y Lourdes Sulca de la Facultad de Ciencias Biológicas de la Universidad Nacional Mayor de San Marcos, Lima; asimismo, al Dr. Julio Manosalva por las facilidades brindadas.

\section{Literatura citada}

Asato, R.; M. Wakuda and T. Sueyoshi. 1991. A case of human infection with Anisakis physeteris larvae in Okinawa, Japan. Jpn. J. Parasitol., 40:181-183.

Barriga, J.; F. Salazar y E. Barriga. 1999. Anisakiasis: presentación de un caso y revisión de la literatura. Rev. Gastroent. Peru., 19:317-323. 
Barrantes, A y C.A. Jara. 1992. Frecuencia de helmintos y crustáceos parásitos de Coryphaena hippurus "dorado", procedente de la zona norte del mar peruano. I Jor Inv Cien Biól 23-24 nov., Trujillo, Perú, pp. 119-122.

Bush, A. O.; K. D. Lafferty; J. M. Lotz and A.W. Shostak. 1997. Parasitology meets ecology on its own terms: Margolis et al. revisited. J. Parasitol., 83:575-583.

Cabrera, R y L. Suárez-Ognio. 2002. Probable emergencia de anisakidosis por larvas de Anisakis physeteris en la costa peruana durante el fenómeno "El Niño" 1997-98. Parasitol. Latinoam., 57 (En prensa).

Cabrera R. Ch y V. M. Tantaleán. 1995. Algunos helmintos de Scomber japonicus "caballa" de la reserva nacional de Paracas. Biotempo., 2:85-86.

Estrella, A. C.; R. Guevara-Carrasco y L. J. 1998. Palacios Informe estadístico de los recursos hidrobiológicos de la pesca artesanal por especies, artes, caletas y meses durante el primer trimestre de 1998. Inf. Inst. Mar. Perú., N. ${ }^{\circ}$ 139: 1-229.

Ishikura, H.; K. Kikuchi; K. Nagasawa; T. Ooiwa; H. Takamiya; N. Sato and K. Sugane. 1992 Anisakidae and anisakidosis. In: T. Sun. (Ed). Prog. Clin. Parasitol., Springer-Verlag, New York, p. 43-102.

Kagei, N.; M. Sano; Y. Takahashi; Y. Tamura; M. Sakamoto. 1978. A case of acute abdominal syndrome caused by Anisakis type-II larva. Jpn. J. Parasitol., 27:427-431.

Kardousha, M.M. 1992. Helminth parasite larvae collected from Arabian gulf fish (coasts of the United Arab Amirates) (I) Anisakid larvae (Nematoda: Anisakidae). Jpn. J. Parasitol., 41:464-472.
Koyama, T.; A. Kobayashi; M. Kumada; Y. Komiya; T. Oshima; N. Kagei; T. Ishii and M. Machida. 1969. Morphological and taxonomical studies on Anisakidae larvae found in marine fishes and squids. Jpn. J. Parasitol., 18:466-468 (Translation fron Japanese by Fisheries Marine Services. Translation Ser N ${ }^{\circ} 3082$, 1974).

Pérez, I.; A. Chávez y E. Casas. 1999. Presencia de formas parasitarias en peces comerciales del mar peruano. Rev. Inv. Vet. Perú., 10:34-38.

Ruelas, N. y E. Córdova. 1993. Estudio de los helmintos de Coryphaena hippurus L. 1758 de la costa sur del Perú. Resúmenes XI Cong. Latinoam. Parasit. y I Cong. Peruano Parasit. 21-26 nov. Lima, Perú. p. 143.

Strømnes, E and K. Andersen. 2000. "Spring rise" of whaleworm (Anisakis simplex; Nematoda, Ascaridoidea) third-stage larvae in some fish species from Norwegian waters. Parasitol. Res., 86:619-624.

Tantaleán, V. M. 1972. La presencia de larvas de Anisakis sp. en algunos peces comerciales del mar peruano. Rev. Per. Med. Trop., UMSM 1:3843.

Tantaleán, V. M and F.A. Huiza. 1993. Nematode larvae with medical importance found in sea fish from the Peruvian shore, with two records of human infections. Rev. Per. Med. Trop., UNMSM 7:61-65.

Tantaleán, V. M.; L. Sarmiento y A. Huiza. 1992. Digeneos (Trematoda) del Perú. Bol. Lima., 14:47-84. 